\title{
ADVERSITÉ FAMILIALE ET TROUBLES DU COMPORTEMENT AU DÉBUT DE LA PÉRIODE DE FRÉQUENTATION SCOLAIRE
}

\author{
FRANK VITARO, \\ RICHARD E. TREMBLAY, \\ et \\ CLAUDE GAGNON \\ Université de Montréal \\ et \\ Groupe de recherche sur l'inadaptation \\ psycho-sociale chez l'enfant (GRIP)
}

\section{RÉSUMÉ}

La présence à la maternelle et en première année des enfants avec un profil comportemental atypique est mise en relation avec le niveau d'adversité familiale des enfants. L'échantillon est composé de 3026 enfants dont les enseignants ont évalué le répertoire de comportement en maternelle et en première année. Trois dimensions de comportement sont considérées lors de ces évaluations : les problèmes d'externalisation (i.e., agressivité-turbulence), les problèmes d'internalisation (i.e., anxiété-retrait social), et des déficits au plan des comportements de prosocialité. Les cas atypiques sont constitués par les enfants qui obtiennent des cotes 1.5 écart-type au-delà de la moyenne des enfants de même sexe au sein de l'échantillon. Les résultats montrent qu'environ deux fois plus d'enfants jugés atypiques en maternelle et en première année sur l'axe des problèmes d'externalisation subissent un niveau élevé d'adversité familiale comparativement à leurs homologues jugés atypiques en maternelle seulement. La discussion porte sur le dépistage des enfants dont le profil comportemental risque de persister au cours de la transition maternelle-première année.

Les études sur les enfants à risque (Watt, Anthony, Wynne, \& Rolf, 1984) montrent que certains facteurs peuvent être associés à l'origine des troubles du comportement dès la naissance, alors que d'autres apparaissent plus tard au cours du développement de l'enfant. Un moment privilégié pour le dépistage des difficultés de comportement est cependant l'entrée dans le système scolaire, et en particulier lors de ce passage important du milieu familial au milieu scolaire (i.e., maternelle). Pour plusieurs enfants, des difficultés importantes de comportement

Cette recherche fut rendue possible grâce à des subventions du Fonds de recherche en santé du Québec et du Conseil de recherche en sciences humaines du Canada. Nous tenons à remercier les enseignants et les parents des enfants de maternelle et de première année qui ont participé à cette fecherche. Nous remercíons egalement Lyse Desmarais-Gervais, Nicole Thériault, Hẻlène Boileau, ct Hélène Beaumont pour leur participation à la collecte et à l'analyse des données. Les demandes d'information ou de tirés-à-part peuvent être adressées au premier auteur. Ecole de psycho-éducation, Université de Montréal, 750 boulevard Gouin est, Montréal, Quẻbec H2C 1 A6. 
se manifestent ou se développent ả la maternelle ou au cours des premières années d'école primaire (Alexander \& Entwistle, 1988; McDermott \& Watkins, 1981; Parker \& Asher, 1987; Rutter, Tizard, \& Whitmore, 1970; Tremblay \& Baillargeon, 1984). Le pronostic du succès scolaire et de l'adaptation sociale de la majorité de ces enfants est très mauvais (Fischer, Rolf, Hasazi, \& Cummings, 1984; Kohn, 1977; Kellam, Granch, Agrawal, \& Ensminger, 1975; Tremblay et al., 1992). En conséquence, il semble justifié de chercher à bien identifier les enfants qui présentent ces risques de mésadaptation scolaire et psycho-sociale en vue de rationnaliser les efforts de prévention ou d'intervention précoce: les enfants en difficulté d'adaptation psychosociale pourraient recevoir plus d'aide que les autres en raison de leurs besoins d'aide particuliers. La maternelle constitue un moment privilégié à cette fin puisque son mandat est clairement la socialisation de l'enfant (Ministère de l'Education du Québec, 1983). Mais encore faut-il être en mesure de distinguer les enfants dont les difficultés d'adaptation s'avéreront stables (i.e., maternelle et première année) de ceux dont les difficultés de comportement sont passagères (i.e., maternelle seulement).

Les données recueillies dans une étude sur le développement de garçons identifiés en maternelle par l'enseignante comme présentant des problèmes d'agressivité et d'hyperactivité (Loeber, Tremblay, Gagnon, \& Charlebois, 1989; Tremblay et al., 1991) montrent que même si ce type de problème de comportement est plutôt stable au cours du développement, il y a un pourcentage d'enfants qui fluctuent d'une année à l'autre, se retrouvant tantôt au-delà, tantôt en deçà du point de coupure déterminant la catégorie des cas extrêmes. Dans leur étude des problèmes d'hyperactivité chez les enfants du primaire de la région d'Ottawa, Trites et Laprade (1983) rapportent également des fluctuations d'une année à l'autre dans la manifestation de comportements hyperactifs: si $90 \%$ des sujets évalués une année en bas du point de coupure sur le Conners' Teacher Rating Scale (Conners, 1969) l'ont été à nouveau l'année suivante, un peu moins de la moitié de ceux qui avaient été évalués au-delà du point de coupure à la première évaluation l'ont été à nouveau l'année suivante. Enfin, O'Donnell, Leicht, Phillips, Marnett, et Horn (1988) ont aussi évalué la stabilité des problèmes de comportement chez les écoliers de la première à la quatrième année. Les problèmes d'agressivité et d'hyperactivité-inattention évalués par les enseignants se sont avérés stables d'une année à l'autre ( $r$ entre .34 et .68). La stabilité des problèmes d'anxiété-retrait social était plus faible. Toutefois, les cas jugés extrêmes (1.5 écart-type au-delả de la moyenne) étaient peu concordants d'une année à recension des écrits sert à illustrer la relative instabilité d'une année à l'autre des enfants jugés atypiques par leur enseignant.

Etant donné que pour la majoritél des enfants au Québec la maternelle est leur première expérience sociale dans un groupe formel de pairs, il est possible que certains utilisent des comportements de retrait social ou d'agressivité comme façon passagère d'expérimenter des rôles sociaux. Pour d'autres, un tel profil comportemental risque de s'avérer persistant et même de s'aggraver à la suite du passage en première année. Certaines variables d'ordre familial peuvent être associées à la persistance, à l'aggravation, ou à la résorption des problèmes d'ajustement social au cours de la période de transition de la maternelle d̀ la
première année. 


\section{L'ENVIRONNEMENT SOCIO-FAMILIAL}

Tremblay et Zhou (1991) ont montré que certaines caractéristiques des parents (surtout celles de la mère) tels la scolarité, l'occupation, et l'âge à la naissance du premier enfant permettaient de prédire les difficultés de comportement à la maternelle. Les résultats de Tremblay (1991) viennent étayer les constatations précédentes: dans un échantillon de garçons de milieux défavorisés, $21,4 \%$ de ceux jugés physiquement agressifs en maternelle proviennent de familles non intactes comparativement à $11,6 \%$ de familles intactes. Aussi, deux fois plus de garçons physiquement agressifs sont enfants uniques comparativement aux autres. Les résultats concernant l'âge de la mère à la naissance des garçons confirment que les enfants de mères adolescentes risquent d'avoir plus de problèmes de comportement que les autres.

La structure de la cellule familiale, le niveau de revenu familial, le régime de travail des parents, leur état de santé mentale, leur niveau éducationnel, ainsi que divers aspects du vécu de l'enfant au sein de sa famille tel la mésentente des parents, la présence de frères ou soeurs ou de grands-parents, des séparations prolongées pour raison d'hospitalisation ou autre, des changements fréquents de domicile ou d'école ont aussi été associés, seuls ou en combinaison, à des problèmes actuels ou futurs d'adaptation à l'école primaire (Boyle \& Offord, 1988; Markman \& Jones-Leonard, 1985; Quinton \& Rutter, 1976; Rutter, Cox, Tupling, Berger, \& Yule, 1975; Schachar \& Wachsmuth, 1991; Schneider, Boucher, Rivest, \& Taillefer, 1984). Certains de ces facteurs (par exemple le statut socioéconomique ou la structure de la cellule familiale) ne sont pas en soi des déterminants des difficultés d'adaptation, mais ils servent d'indicateurs d'un ensemble de facteurs de stress qui, eux, ont un impact sur le comportement quotidien de l'enfant (Garbarino, 1982). D'autres aspects, par exemple la discorde maritale, ont par contre un effet plus direct tel qu'ont tenté de le démontrer Markman et Jones-Leonard (1985). Toutefois, peu d'auteurs se sont préoccupés de vérifier si divers éléments d'adversité familiale sont associés à la persistance des problèmes de comportement. La présente recherche se propose de combler cette lacune en tentant d'établir un lien entre le niveau d'adversité socio-familiale et la persistance des problèmes de comportement de la maternelle à la première année.

Rutter (1985) a analysé les effets simples et combinés de certaines variables de risque apparentées à celles qui sont exposées ici. La présence d'une seule variable de risque ne semble pas augmenter la probabilité de problèmes ultérieurs d'adaptation. Par contre, les risques quadruplent lorsque deux variables de risque sont simultanément présentes. La présence de trois ou quatre d'entre elles augmente considérablement les risques d'inadaptation. De leur côté, Schneider et al. (1984) rapportent une corrélation positive entre le nombre de variables de risque présentes dans l'histoire personnelle et sociale d'enfants et un diagnostic de troubles de comportement ou d'apprentissage. Ces facteurs de risque sont: une institutionnalisation de l'enfant, des problèmes maritaux, une séparation de l'enfant de ses parents, la psychopathologie chez les parents, les complications durant la grossesse, une naissance prématurée, le faible poids à la naissance, l'hospitalisation au cours de la jeune enfance, et les fréquents changements d'adresse. Enfin, Kelso et Stewart (1986) ont constaté que le nombre d'indicateurs (i.e., fréquence et variété des comportements agressifs ou antisociaux, 
âge d'apparition de ces comportements, tendance à mettre le feu, et déviance dans la famille) plutôt que leur nature propre permet de distinguer (et donc de prédire) les garçons âgés de 5 à 14 ans dont les désordres de la conduite persistent de ceux dont les problèmes se résorbent.

Ces résultats militent en faveur de la constitution d'une cote composite d'adversité familiale qui tiendrait compte d'un ensemble de facteurs de risque. Cette avenue est celle retenue dans la présente recherche. La cote composite d'adversité familiale réunit diverses variables de risque (définies plus loin) et est représentée sous la forme d'un indice numérique.

\section{LES PROBLÈMES D'EXTERNALISATION ET D'INTERNALISATION}

Deux grandes catégories de problèmes de comportement sont considérées dans cette étude : les comportements d'internalisation (retrait social-anxiété) et les comportements d'externalisation (agressivité-turbulence) (Achenbach \& Edelbrock, 1981; Quay, 1979). La classification internalisation - externalisation correspond à la structure de base des comportements sociaux approche-fuite (Laborit, 1979; Masters, 1979) et à la capacité qu'ont les adultes et les pairs de juger le comportement des enfants avec lesquels ils interagissent. Il est extrêmement difficile pour des enseignants, par exemple, de différencier les enfants hyperactifs des enfants agressifs avant l'âge de 7-8 ans (voir Fowler \& Park, 1979; Schachar, Rutter, \& Smith, 1981; Trites \& Laprade, 1983). Ces différenciations doivent être faites par des procédures d'observation directe et l'analyse détaillée de nombreux facteurs une fois le premier dépistage effectué (Milich, Loney, \& Landau, 1982; Rutter et al., 1970). Par contre, il est possible de bien distinguer les comportements prosociaux des comportements agressifs (Milich \& Landau, 1984; Ross, Lacey, \& Parton, 1965; Weir \& Duveen, 1981). Cette différenciation peut être fort utile dans une perspective de prédiction. Il est probable que la présence ou l'absence de comportements prosociaux chez un enfant qui présente des difficultés de comportements (e.g., agressivité) agisse comme facteur de protection ou d'aggravation par rapport à ces difficultés (Campbell \& Cluss, 1982; Eron \& Huesmann, 1983; Tremblay, Vitaro, Gagnon, Piché, \& Royer, sous presse).

\section{Objectifs}

Le premier objectif de la présente recherche consiste à comparer des groupes d'enfants dont le profil de comportement (établi selon les trois axes décrits précédemment) est jugé extrême à la maternelle et en première année (i.e., cas stables) ou à la maternelle seulement (i.e., cas transitoires). Le second objectif consiste à vérifier si la proportion de cas extrêmes à la maternelle est reliée à des variables d'ordre socio-familial. Enfin, le troisième objectif consiste à vérifier si la proportion de cas extrêmes stables est reliée à des variables d'ordre sociofamilial.

Tel que signalé précédemment, la stabilité des cas extrêmes selon le point de vue des enseignants est plutôt modérée. Lorsque les points de coupure sont relativement sévères (i.e., 1.5 écart-type au-delà de la moyenne), il arrive que la proportion de cas extrêmes stables soit même faible (O'Donnell et al., 1988). 
Toutefois la majorité des études pêche par un nombre restreint de cas extrêmes, d'où une difficulté importante pour l'étude de leur stabilité. Par conséquent, l'étude de la stabilité des cas extrêmes (i.e., 1.5 écart-type au-delà de la moyenne) et des facteurs d'ordre socio-familial qui y sont associés commande un vaste échantillon de départ en raison de l'attrition inévitable et de la faible stabilité des cas extrêmes. Ce problème est surmonté dans la présente étude puisque l'échantillon de départ comporte un nombre élevé d'enfants des deux sexes. Le critère retenu dans cette étude pour définir les cas extrêmes est un critère statistique, soit 1.5 écart-type au delà de la moyenne propre à chaque sexe sur chaque dimension comportementale. Ce critère statistique comporte deux avantages: il a été utilisé par d'autres auteurs pour définir les cas extrêmes et il correspond approximativement au 90ième percentile de la distribution des cotes. Or, selon certains auteurs (Achenbach \& Edelbrock, 1981), le 90ième percentile constitue un critère significatif pour définir les cas cliniques dans les études épidémiologiques.

Par ailleurs, le sexe de l'enfant s'est révélé systématiquement associé à l'incidence des difficultés de comportement à l'école. Chez les filles, l'incidence des difficultés de comportements est plus faible que chez les garçons (Achenbach \& Edelbrock, 1978; Rutter et al., 1970; Tremblay \& Baillargeon, 1984; Werner \& Smith, 1982). En retour, les problèmes ultérieurs d'adaptation pour les garçons et les filles avec des problèmes de comportement sont également sérieux quoique partiellement distincts (Robins, 1966, 1986). Les analyses relatives aux garçons seront donc distinctes de celles relatives aux filles.

\section{MÉTHODOLOGIE}

\section{Échantillon}

Un échantillon représentatif des enfants fréquentant les classes de maternelle dans les écoles publiques du Québec en 1986-87 fut constitué par sélection au hasard des commissions scolaires au sein de chacune des 11 régions administratives de la province. Un nombre proportionnel d'enfants fut ensuite sẻlectionné par hasardage au sein de chaque commission scolaire. A cet effet, un programme informatisé a été appliqué par le ministère de l'Education du Québec à la liste des enfants des commissions scolaires choisies.

L'échantillon initial comprenait 6397 enfants en maternelle (T1). Des informations de la part des éducatrices de maternelle ont été obtenues pour 4378 enfants, soit un taux de réponse de $68,4 \%$. Une relance a été effectuée l'année suivante pour tous les enfants de l'échantillon initial. Les enfants étaient alors en première année (T2). A l'occasion de cette relance, des informations de la part de l'enseignant titulaire sont obtenues pour 4050 enfants, soit un taux de réponse de $63,3 \%$.

Des informations de la part des enseignants titulaires sont disponibles à $\mathrm{T} 1$ et T2 pour 3026 enfants (1 538 garçons et 1488 filles), soit $47,3 \%$ de l'échantillon initial. Les parents ont également fourni des informations à T1 pour 4057 enfants. Quatre-vingt-seize pourcent des enfants de l'échantillon final sont de langue maternelle française. Un peu plus des trois quarts $(77,7 \%)$ des enfants vivent avec leurs deux parents. Près de $10 \%(8,9 \%)$ vivent avec leur mère alors que $3,8 \%$ vivent avec leur mere et un substitut paternel. Les autres vivent avec 
leur père $(1,3 \%)$, une mère adoptive $(0,8 \%)$, les grand-parents $(0,1 \%)$, ou quelqu' un d'autre $(1,1 \%)$. Enfin, aucune information n'est disponible relativement à la structure de la cellule familiale pour $6,4 \%$ des enfants.

Une analyse multivariée de la variance indique que le comportement des enfants présents dans l'échantillon à T1 mais absents à T2 (soit 695 garçons et 609 filles) est significativement différent aux yeux des éducatrices à T1 $(F(3$, $4321)=4.67, p<.01)$. Cette différence est indépendante du sexe des enfants. Des analyses de la variance subséquentes révèlent que cette différence se limite toutefois aux comportements prosociaux. Les enfants présents à T1 seulement sont significativement moins prosociaux que ceux présents à $\mathrm{T} 1$ et à $\mathrm{T} 2(M=6.82$ vs $7.28 ; F(1,4323)=11.82, p<.01)$.

\section{Instruments}

Le questionnaire d'évaluation du comportement. Les enseignants de maternelle et de première année sont invités à remplir pour chaque enfant de leur classe qui fait partie de l'échantillon, le Questionnaire d'évaluation des comportements sociaux (QECS). Il s'agit de la version française du Preschool Behavior Questionnaire (Behar \& Stringfield, 1974; Fowler \& Park, 1979) jumelé au Prosocial Behavior Questionnaire (Weir \& Duveen, 1981).

La fidélité et la validité de ces deux instruments ont été établies par plusieurs groupes de chercheurs (Behar \& Stringfield, 1974; Campbell \& Cluss, 1982; Hoge, Meginbir, Khan, \& Wheatherall, 1985; Rubin \& Clark, 1983; Rubin, Moller, \& Emptage, 1986; Rutter, 1967; Weir \& Duveen, 1981). Fowler et Park (1979) aux Etats-Unis, Hoge et al. (1985) au Canada-anglais, et Tremblay, DesmaraisGervais, Gagnon, et Charlebois (1987) au Canada-français ont démontré que deux facteurs stables pouvaient être extraits du Preschool Behavior Questionnaire: les comportements d'agressivité-turbulence (externalisation) et les comportements d'anxiété-retrait social (internalisation). L'analyse factorielle des données obtenues suite à une intégration de 10 items du Prosocial Behavior Questionnaire au Preschool Behavior Questionnaire (constituant ainsi le QECS) pour un échantillon de 1161 garçons en maternelle à Montréal, a permis d'obtenir les deux facteurs "agressivité-turbulence» (13 items) et «anxiété-retrait social» (six items), ainsi qu'un troisième facteur "comportements prosociaux" (10 items) (Tremblay, Vitaro, Gagnon, Piché, \& Royer, sous presse).

La version préscolaire de ce questionnaire peut être utilisée jusqu'en deuxième année du primaire (Rubin et al., 1986). L'enseignant indique sur une échelle à trois unités si chaque brève description de comportement ne s'applique pas, s'applique parfois, ou s'applique souvent à l'enfant dont le comportement est évalué. Le total pour les 13 items d'agressivité-turbulence peut varier de 0 à 26. Celui de l'échelle anxiété-retrait social peut varier de 0 à 12 et celui pour l'échelle de prosocialité de 0 à 20 .

Informations relatives au milieu familial. Les mères sont invitẹes à T1 et à T2 à fournir les informations suivantes: la structure de la cellule familiale (biparentale, monoparentale, reconstituée, institution), le niveau d'éducation de chaque parent ou du parent avec qui l'enfant vit, l'emploi occupé par l'un et/ou l'autre parent et, enfin, l'age de chaque parent à la naissance de l'enfant de l'étude. 
A l'instar de Tremblay et al. (1991) un indice d'adversité familiale a été constitué en faisant la moyenne des poids 0 ou 1 accordés aux sept variables sociodémographiques précédentes. Une moyenne est calculée lorsque des informations sont disponibles pour au moins quatre variables. ${ }^{2}$ Tel que déjà souligné, ces variables se sont avérées associées dans d'autres études à la présence de problèmes d'adaptation sociale chez l'enfant. La structure de la cellule familiale obtient une cote 0 pour les cas où l'enfant vit avec ses deux parents naturels et une cote 1 pour tous les autres cas. Les mesures sur les six autres variables obtiennent une cote 0 ou 1 suivant qu'elles se situent ou non au-delà du $25^{\star}$ percentile de leur distribution respective. L'échelle de prestige occupationnel de Blishen et McRoberts (1976) sert d̀ situer l'emploi de chaque parent sur une échelle continue.

Un peu plus de la moitié des enfants de l'échantillon $(52,7 \%)$ obtiennent une cote moyenne d'adversité entre 0 et .24 (niveau faible) alors qu'un autre tiers $(34,4 \%$ ) obtiennent des cotes entre .28 et .56 (niveau moyen). Enfin, $12,9 \%$ des enfants ( $13,6 \%$ des garçons et $12 \%$ des filles) obtiennent une cote supérieure à .57 (niveau élevé).

\section{Procédure}

Après avoir repéré par la banque informatisée du ministère de l'Education l'école que fréquente chacun des enfants à évaluer, nous avons envoyé au mois de mars de chaque année le QECS aux enseignants concernés. L'enseignant nous retourne les questionnaires lorsqu'ils sont complétés. Les parents reçoivent par l'entremise de l'enseignant et de leur enfant les questionnaires qui leur sont destinés (i.e., informations relatives au milieu familial). Après l'avoir rempli, ils le retournent à l'école. L'enseignant nous retourne les questionnaires de tous les enfants de sa classe une fois complétés.

\section{RÉSULTATS}

\section{Stabilité des évaluations comportementales}

Les résultats rapportés dans cette section concernent les 3026 enfants de l'échantillon final pour lesquels des données sont disponibles à T1 et à T2; les nombres varient légèrement selon les variables ou la source d'évaluation considérées (i.e., enseignants et/ou parents) en raison de données manquantes.

Catégories extrêmes. Les premières analyses concernent les enfants des deux sexes qui ont obtenu à T1, à l'une ou l'autre échelle du QECS, une cote supérieure d'un écart-type et demi à la moyenne des enfants de même sexe. Tel qu'illustré au tableau 1, les garçons ayant obtenu une cote extrême sur l'axe agressivitéturbulence représentent $11,1 \%$ de la tranche masculine de l'échantillon $(M=16,47)$. Les filles jugées atypiques sur cette même dimension (selon le critère précédent) représentent $9,4 \%$ de la tranche féminine de l'échantillon $(M=11.68)$.

Au niveau de l'échelle anxiété-retrait, $12,2 \%$ des garçons $(M=7.33)$ et $14,1 \%$ des filles $(M=6.46)$ obtiennent à T1 une cote supérieure de 1.5 écart-type à la moyenne propre aux enfants de leur sexe au sein de l'échantillon final. Enfin, $11,5 \%$ des garçons $(M=8)$ et $17 \%$ des filles $(M=1.41)$ sont jugés peu prosociaux à T1 (i.e., obtiennent une cote à l'échelle de prosocialité inférieure de 1.5 
écart-type par rapport à la moyenne des enfants de leur sexe). De 16 à $22 \%$ des garçons jugés extrêmes sur une échelle le sont aussi sur au moins une autre échelle. Chez les filles, les pourcentages varient de 21 à $32 \%$.

Afin de bien situer les cotes des cas jugés extrêmes par les enseignants sur chaque échelle du QECS, il faut se rapporter aux moyennes et aux écarts-type présentés au tableau 2. Ainsi, les garçons et les filles jugés extrêmes sur l'échelle agressivité-turbulence ont des cotes autour du point milieu de l'échelle. Répétant l'exercice précédent pour les échelles d'anxiété-retrait et de prosocialité, il est possible de constater que les garçons jugés extrêmes se situent au-delả du point milieu sur l'échelle anxiété-retrait et tout près du minimum sur l'échelle de prosocialité. A l'instar des garçons, les filles jugées extrêmes sur ces deux mêmes échelles obtiennent des cotes légèrement au-delà du point milieu sur l'échelle anxiété-retrait et quasi-nulles sur l'échelle prosocialité.

\section{Catégories extrêmes et adversité familiale}

A T1, 9,9\% des garçons et $8 \%$ des filles avec un niveau d'adversité faible (i.e., 0 à 3 éléments d'adversité = cote de 0 à .56) sont jugés extrêmes sur l'axe agressivité-turbulence comparativement à 14,8 \% des garçons et 13,1\% des filles avec un niveau d'adversité élevé (i.e., quatre éléments et plus d'adversité = cote de .57 à 1). L'interaction entre le nombre de garçons ou de filles extrêmes à T1 sur l'axe agressivité-turbulence et le niveau d'adversité socio-familiale est statistiquement significative, chi-carré( 1$)=3.86$ pour les garçons et 4.14 pour les filles, $p<.05$. En retour, la proportion d'enfants jugés extrêmes à T1 sur les dimensions anxiété-retrait et prosocialité ne varie pas significativement $(p>.05)$ selon le niveau d'adversité socio-familiale (garçons extrêmes : $11,4 \%$ avec niveau faible vs $13,8 \%$ avec niveau élevé pour anxiété-retrait et $11,5 \%$ avec niveau faible vs $9,7 \%$ avec niveau élevé pour prosocialité; filles extrêmes: $13,3 \%$ avec niveau faible vs $18,3 \%$ avec niveau élevé pour anxiété-retrait et $16,8 \%$ avec niveau faible vs $20,5 \%$ avec niveau élevé).

\section{Stabilité des catégories extrêmes}

La prochaine section s'intéresse à la stabilité de T1 à T2 des catégories extrêmes établies à T1. Les critères définissant les catégories extrêmes à T2 sont les mêmes que ceux ayant présidé à la constitution des catégories extrêmes à T1. A l'examen du tableau 1 , il ressort que près de la moitié $(48,5 \%)$ des garçons jugés extrêmes à T1 sur l'échelle agressivité-turbulence sont jugés de la même manière à T2. Ces sujets composent le groupe des cas stables. La proportion de filles jugées extrêmes à $\mathrm{T} 1$ et à $\mathrm{T} 2$ sur cette même dimension est de $37,4 \%$. Les proportions de garçons et de filles stables sur l'échelle agressivité-turbulence sont significativement supérieures aux proportions attendues au hasard en vertu des critères de classification retenus et des pourcentages de cas extrêmes sur cette dimension à T2 $(z=15.96$ pour les garçons et 10.79 pour les filles, $p<.01)$. Le nombre des enfants jugés extrêmes à $\mathrm{T} 1$ et à $\mathrm{T} 2$ ou à $\mathrm{T} 1$ seulement (cas transitoires) est présenté au tableau 1. La moyenne des garçons extrêmes stables sur cette échelle est de 17.04 à $\mathrm{T} 1$ et de 17.42 à T2 $\left(E . T_{*}=3.53\right.$ et 3.76$)$. Celle des filles jugées extrêmes stables est de 12.59 à $\mathrm{T} 1$ et de 14.69 à T2 $(E . T .=4.33$ et 4.65$)$. 
La proportion de cas stables sur l'échelle anxiété-retrait est inférieure à la proportion de cas stables sur l'axe agressivité-turbulence (voir le tableau 1; $z=3.14$ pour les garçons et 5.18 pour les filles, $p<.05$ ). Ainsi $30,3 \%$ des garçons et $25,7 \%$ des filles jugés extrêmes à T1 sur l'échelle anxiété-retrait le sont encore à T2 (cas stables). Quoique modestes, les proportions de cas stables sur l'échelle anxiété-retrait social sont supérieures au hasard $(z=8.60$ pour les garçons et 5.0 pour les filles, $p<.05$ ). La moyenne des garçons jugés extrêmes stables sur cette dimension est de 7.82 à $\mathrm{T} 1$ et de 7.79 à $\mathrm{T} 2(E . T .=1.81$ et 1.64$)$

\section{TABLEAU 1}

Nombre de cas extrêmes à $\mathrm{T} 1$ seulement et à $\mathrm{T} 2$

pour chaque échelle d'évaluation et selon le sexe

\begin{tabular}{|c|c|c|c|c|c|c|c|}
\hline \multirow{4}{*}{$\begin{array}{l}\text { Echelle } \\
\text { Agressivité- } \\
\text { turbulence } \\
(>\bar{x}+1.5 S D)\end{array}$} & \multirow{3}{*}{$\begin{array}{c}\text { Sexe } \\
\text { garçons }\end{array}$} & \multicolumn{6}{|c|}{ Cas extrêmes } \\
\hline & & \multicolumn{2}{|c|}{ à $\mathrm{T1}$} & \multicolumn{2}{|c|}{$\begin{array}{l}\text { à } \mathrm{T} 1 \text { seulement } \\
\text { (cas transitoires) }\end{array}$} & \multicolumn{2}{|c|}{$\begin{array}{l}\text { à } \mathrm{T} 1 \text { et à } \mathrm{T} 2 \\
\text { (cas stables) }\end{array}$} \\
\hline & & $171 / 1542$ & $(11,1 \%)$ & 88 & $(51,5 \%)$ & 83 & $(48,5 \%)$ \\
\hline & filles & $139 / 1486$ & $(9,4 \%)$ & 87 & $(62,6 \%)$ & 52 & $(37,4 \%)$ \\
\hline \multirow{2}{*}{$\begin{array}{l}\text { Anxiété- } \\
\text { retrait social } \\
(>\bar{X}+1.5 S D)\end{array}$} & garçons & $189 / 1543$ & $(12,2 \%)$ & 128 & $(67,7 \%)$ & 61 & $(32,3 \%)$ \\
\hline & filles & $219 / 1489$ & $(14,1 \%)$ & 156 & $(74,3 \%)$ & 54 & $(25,7 \%)$ \\
\hline \multirow{2}{*}{$\begin{array}{l}\text { Prosocialité } \\
(<\bar{x}-1.5 S D)\end{array}$} & garçons & $177 / 1534$ & $\left(11,5 \%_{0}\right)$ & 138 & $\left(78,0 \sigma_{0}\right)$ & 39 & $(22,0 \%)$ \\
\hline & filles & $252 / 1481$ & $(17,0 \%)$ & 181 & $(71,8 \%)$ & 71 & $(28,2 \%)$ \\
\hline
\end{tabular}

TABLEAU 2

Moyennes et écarts-type obtenus à $\mathrm{T} 1$ et à $\mathrm{T} 2$

pour les enfants de tout l'échantillon à chaque échelle du QECS

\begin{tabular}{lccccccc}
\hline & & \multicolumn{5}{c}{ Echelles du QECS } \\
\cline { 3 - 8 } Année & Sexe & $\begin{array}{c}\text { Agressivité- } \\
\text { turbulence } \\
\text { (min. 0, max. 26) }\end{array}$ & $\begin{array}{c}\text { Anxiété- } \\
\text { retrait social } \\
\text { (min. 0, max. 12) }\end{array}$ & $\begin{array}{c}\text { Prosocialité } \\
\text { (min. 0, max. 20) }\end{array}$ \\
\hline T1 & garçons & $4.60^{\mathrm{a}}$ & $(5.55)^{\mathrm{b}}$ & 2.38 & $(2.43)$ & 6.35 & $(4.46)$ \\
T2 & filles & 2.35 & $(3.74)$ & 2.02 & $(2.26)$ & 8.21 & $(4.53)$ \\
& garçons & 4.57 & $(5.54)$ & 2.88 & $(2.53)$ & 6.20 & $(4.37)$ \\
\hline $\begin{array}{l}\text { a: moyenne } \\
\text { b: écart-type }\end{array}$ & filles & 2.41 & $(3.97)$ & 2.66 & $(2.52)$ & 7.58 & $(4.53)$ \\
\hline
\end{tabular}


alors que celle des filles jugées stables est de 7.0 à $\mathrm{T} 1$ et de 7.54 à T2 $(E . T,=1.84$ et 1.41 ).

Au regard de l'échelle de prosocialité, $22,0 \%$ des garçons et $28,2 \%$ des filles jugés non prosociaux à T1 sont jugés de la même manière à T2 (voir le tableau 1). A nouveau ces proportions sont inférieures aux proportions de cas stables sur l'échelle agressivité-turbulence $(z=5.18$ pour les garçons, $p<.05$, et 1.88 pour les filles, $p=.06$ ). Malgré tout, les proportions des cas stables sur l'axe de non prosocialité ne sont pas une question de hasard $(z=4.68$ pour les garçons et 5.87 pour les filles, $p<.05$ ). La moyenne des 39 garçons jugés extrêmes sur cette échelle à $\mathrm{T} 1$ est de $0(E . T .=0)$. Leur moyenne à T2 est aussi égale à 0 avec un $E . T .=0$. La moyenne des filles jugées extrêmes stables sur cette échelle est de 1.18 à T1 et de .91 à T2 $(E . T .=1.19$ et .88$)$.

Les prochaines analyses ont pour but de vérifier si les cas stables se distinguent à T1, d'abord, et à T2 ensuite, au niveau des cotes au QECS. Il est en effet possible que les cas stables affichent à T1 un répertoire de comportement plus détérioré que les cas transitoires. Cette possible différence initiale pourrait expliquer leur plus grande stabilité. Deux analyses multivariées de la variance à un facteur (cas stables et transitoires) ne révèlent aucune différence significative à T1 entre les garçons agressifs-turbulents stables et les garçons agressifs-turbulents transitoires au niveau des trois échelles du QECS. A T2, par définition, les garçons agressifs-turbulents stables $(M=17.42, E . T .=3.76)$ se distinguent de leurs homologues transitoires $(M=6.24, E . T .=4.04)$ au niveau des cotes d'agressivité-turbulence, $F(1,86)=180.97, p<.001$. Une série de tests $t$ pour échantillons reliés permet de constater que les cotes d'agressivité-turbulence des cas transitoires diminuent de T1 à T2 alors qu'il n'y a pas de différence de T1 a T2 pour les cas stables. Les résultats de l'analyse de la variance pour les filles agressives-turbulentes sont semblables à ceux pour les garçons. A T1, il n'y a pas de différence significaive entre les filles agressives-hyperactives stables et leurs consoeurs transitoires au niveau des cotes aux trois échelles du QECS. Toutefois, à $T 2$, les premières $(M=14.69, E . T .=4.64)$ se distinguent des secondes $(M=3.00$, E. $T .=2.81)$ au niveau des cotes d'agressivité-turbulence, $F(1,71)=179.61, p<.001$. Une série de tests $t$ indique que les cotes d'agressivité-turbulence des fillettes transitoires diminuent de T1 à T2 $(p<.05)$ alors que celles des fillettes stables ne changent pas.

Les résultats pour les dimensions anxiété-retrait et nonprosocialité vont dans le même sens que les résultats précédents: il n'y a pas de différence à TI entre les cas stables et les cas non stables. La différence à T2 est toutefois significative, pour les garçons comme pour les filles. Pour les deux dimensions en cause, le profil des cas transitoires s'améliore de T1 à T2 alors que celui des cas stables ne change pas.

\section{Stabilité des catégories extrêmes et adversité familiale}

Dans la dernière série d'analyses, la stabilité des cas extrêmes sur chaque échelle du QECS est mise en relation avec le niveau d'adversité familiale. Comme précédemment, les cotes d'adversité familiale ont été dichotomisées: 0 à 3 éléments d'adversité (cotes moyennes de 0 à .56) vs 4 éléments et plus (cotes de .57 
et plus) d'adversité; ceci a permis d'établir un niveau faible et un niveau élevé d'adversité.

Les résultats indiquent qu'il y a $28,4 \%$ des garçons et $38,1 \%$ des filles extrêmes à T1 et à T2 sur l'échelle agressivité-turbulence du QECS (v.g., cas stables) qui subissent un niveau élevé d'adversité comparativement à $16,7 \%$ des garçons et à 15,2\% des filles jugés extrêmes à T1 seulement (v.g., cas transitoires). Chez les filles, la proportion de cas stables avec un indice d'adversité élevé est significativement plus grande $(z=5.14, p<.01)$ que la proportion de cas transitoires avec un niveau élevé d'adversité familiale. Chez les garçons, la différence entre la proportion de cas stables avec un indice d'adversité élevé et la proportion de cas transitoires dans la même situation est près du seuil de signification ( $z=1.75, p=.08$ ). Les résultats sont illustrés à la figure 1. Par ailleurs, la relation entre le niveau d'adversité familiale et la stabilité des cas extrêmes est près du seuil de signification pour les garçons (chi-carré) $(1)=3.00, p=.08$ ) et au-delà du seuil de signification pour les filles (chi-carré $_{(1)}=8.09, p<.01$ ).

En revanche, la stabilité des cas extrêmes sur les dimensions anxiété-retrait et prosocialité est indépendante du niveau d'adversité familiale.

\section{FIGURE 1}

Pourcentage de cas extrêmes stables et transitoires sur l'échelle agressivitế-turbulence avec un niveau élevé d'adversité familiale

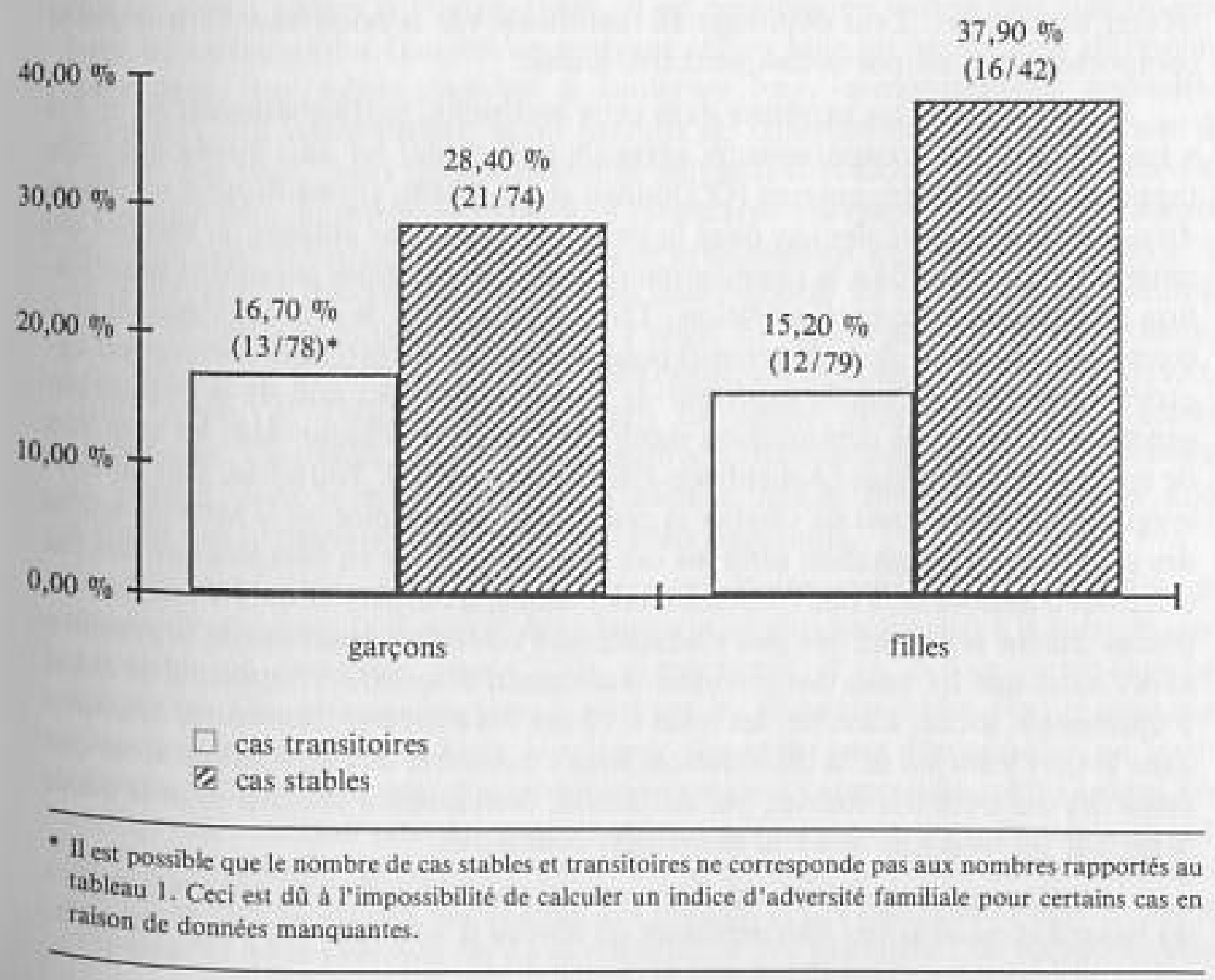




\section{DISCUSSION}

Le premier objectif de cette recherche concerne la stabilité de la maternelle à la première année des enfants jugés extrêmes sur divers axes comportementaux. Le second objectif se rapporte au lien entre le statut de cas extrême à la maternelle et le niveau d'adversité socio-familiale. Enfin, le dernier objectif se préoccupe du lien entre la stabilité du statut de cas extrême de la maternelle à la première annèe et le niveau d'adversité socio-familiale.

Près de la moitié des garçons et plus du tiers des filles jugés extrêmes à T1 sur l'axe des comportements agressifs-turbulents appartiennent encore au groupe des cas extrêmes à T2. Ces proportions importantes d'enfants agressifs-turbulents stables ne sont pas le fruit du hasard. Ces résultats corroborent ceux d'autres auteurs quant à la stabilité des comportements agressifs-turbulents-antisociaux chez les enfants et les adolescents de sexe masculin (Loeber, 1982; Olweus, 1984) et de sexe féminin (Robins, 1986). Les proportions de cas extrêmes stables sur les dimensions anxiété-retrait et prosocialité varient d'un peu plus du cinquième à un peu moins du tiers, selon l'échelle et le sexe. Quoique plus modestes que les proportions d'enfants agressifs-turbulents stables, les proportions d'enfants anxieuxretirés ou non prosociaux stables ne se distinguent pas moins du simple hasard. Malgré tout, ces résultats révèlent une grande plasticité des comportements d'anxiété-retrait et de prosocialité chez les enfants de cet âge, ou encore une grande variabilité dans la façon de les percevoir. Par ailleurs, les cas extrêmes stables ne représentent pas des cas plus sévères à $T 1$ puisqu'il n'y a pas de différence à T1 sur les échelles du QECS entre les cas extrêmes stables et les cas extrêmes transitoires. Leur dépistage en maternelle sur la seule base de leur profil comportemental est par conséquent très limité.

La stabilité des cas extrêmes dans cette recherche, particulièrement en ce quí a trait à l'axe des comportements agressifs-turbulents, est plus élevée que celle rapportée par d'autres auteurs (O'Donnell et al., 1988), probablement en raison du nombre plus élevé des cas dans la présente étude. Par ailleurs, la sévérité des critères qui ont prèsidé à la constitution des groupes extrêmes garantit la signification clinique de cette catégorisation. Tel que déjà noté, le critère retenu ici $(1.5$ écart-type au-dessus de la moyenne) pour définir les cas extrêmes correspond approximativement au décile supérieur de la distribution au sein de la population générale, un point de démarcation significatif au plan clinique dans les enquêtes de type épidémiologique (Achenbach \& Edelbrock, 1981). Toutefois, seul un suivi longitudinal permettrait de vérifier la plus grande fréquence ou la sévérité accrue des problèmes d'adaptation pour les cas extrêmes stables en comparaison des cas extrêmes transitoires. Pour l'instant il est possible de constater qu'à T2 les cas extrêmes stables semblent très peu s'adapter aux nouvelles exigences de la première année alors que les cotes des premiers témoignent d'un effort important et réussi d'ajustement social. En effet, les cotes à T2 des cas extrêmes transitoires se situent dans le tiers mitoyen de la distribution pour l'ensemble de l'échantillon alors que celles des cas extrẻmes stables, par définition, continuent à se situer dans le décíle supérieur ou inférieur selon la dimension considérée.

Les cas extrêmes stables sur l'axe agressivité-turbulence ne sont pas le fruit du hasard et ne sont pas indépendants du niveau d'adversité familiale. En effet, 
près de deux fois plus de garçons et au-delà de deux fois plus de filles extrêmes stables sur l'axe agressivité-turbulence ont un indice élevé d'adversité familiale comparativement à leurs homologues transitoires. A la maternelle, une plus grande proportion d'écoliers et d'écolières avec un niveau d'adversité élevé se trouvent dans le groupe des enfants agressifs-turbulents en comparaison des enfants avec un niveau d'adversité faible. Ces résultats corroborent ceux d'autres recherches (Bouchard, 1981; Garbarino, 1982; Tremblay, Vitaro, Gagnon, \& Perron, 1989). Toutefois, en raison du taux de réponse modéré, ces résultats ne peuvent pas être généralisés ả l'ensemble des enfants de l'échantillon initial, luimême représentatif des enfants québécois du même âge. Ils soulignent néanmoins l'importance de tenir compte de l'adversité socio-familiale dans le dépistage des enfants vulnérables et la mise sur pied de programmes de prévention ou d'intervention précoce. Les problèmes d'internalisation et les comportements prosociaux semblent toutefois indépendants du niveau d'adversité au sein de la famille.

Les raisons pour lesquelles la présence et la persistance des problèmes d'agressivité-turbulence rapportés par les professeurs sont sensibles au niveau d'adversité socio-familiale sont de deux ordres. Il est possible, en premier lieu, que les professeurs réagissent différemment aux enfants de milieu moins favorisé et à ceux de milieu plus favorisé, évaluant plus sévèrement les comportements agressifs-turbulents des premiers que des seconds. Cette attitude discriminatoire s'exercerait particulièrement au chapitre des comportements agressifs-turbulents en raison de l'effet hautement pertubateur de ces comportements en classe et de la relative difficulté pour les enseignants de les gérer efficacement (Coleman \& Gilliam, 1983; Meijer \& Foster, 1989). Il est possible, en second lieu, que les enfants de milieu moins favorisé apprennent moins bien ou apprennent différemment dans leur milieu familial à contrôler leurs comportements agressifsturbulents. En conséquence, leurs patrons de comportement se généralisent à l'école. De plus, leurs comportements agressifs-turbulents sont possiblement moins sensibles au mode de gestion du professeur comparativement aux enfants de milieu plus favorisé. Ces derniers sont possiblement exposés à des règles et à des exemples de conduite ainsi qu'à des contingences de renforcement-punition plus systématiques et plus apparentées à celles en vigueur dans le milieu scolaire. Les travaux de Patterson et de ses collaborateurs (Patterson, 1982; Patterson, Reid, Jones, \& Conger, 1975) offrent un appui indirect aux spéculations précedentes. Toutefois, une observation directe des contingences de renforcement en vigueur dans le milieu scolaire et dans le milieu familial est requise afin d'étayer ces hypothèses explicatives au plan empirique.

En conclusion, les problèmes graves d'externalisation des enfants de maternelle risquent de s'avérer plus nombreux et plus persistants à la suite de leur transition en première année $s$ 'ils proviennent d'un milieu socio-familial défavorisé. Ces enfants devraient constituer les clientèles-cibles des programmes d'intervention précoce destinés à prévenir des problèmes d'adaptation au cours primaire. Ces programmes d'intervention devraient comprendre, entre autres, des ateliers de formation pour les parents et les enseignants au sujet du mode de gestion des problèmes de comportement. Cette suggestion repose sur la possibilité que les modes de gestion déficitaires des parents et des enseignants soit en partie responsable de la présence ou de la persistance des problèmes de comportement 
chez ces enfants. Les programmes d'intervention devraient aussi comprendre des ateliers d'entraînement aux habiletés sociales et aux stratégies de résolution de problèmes.

Plusieurs programmes d'entraînement aux habiletés parentales et/ou aux habiletés interpersonnelles pour l'enfant ont été expérimentés et évalués (Dumas, sous presse; Forehand \& McMahon, 1981; Patterson, Cobb, \& Ray, 1983; Tremblay et al., sous presse). Toutefois, certains auteurs ont souligné les difficultés de recrutement, les problèmes d'attrition, le risque d'échec, et l'effritement rapide des gains réalisés en cours de formation lorsque, précisément, les parents concernés sont désavantagés au plan socio-économique (Dumas, sous presse; Kazdin, 1987). Ces difficultés particulières constituent les défis auxquels les recherches futures devront se confronter.

\begin{abstract}
The aims of this research were twofold: to investigate the relationship between family adversity (based on a composite index) and the number of teacher-rated behaviourally disordered children in kindergarten and to examine the relationship between family adversity and the stability of behavioural disorders from kindergarten to grade one. The sample was comprised of 3,026 children who were rated by their kindergarten and grade one teachers on three behavioural dimensions (aggressiveness-disruptiveness13 items; anxiety-withdrawal -6 items; prosociality -10 items) using the Preschool Behavior Questionnaire (Behar \& Stringfield, 1974) and the Prosocial Behavior Questionnaire (Weir \& Duveen, 1981). The Family Adversity Index (FAI) was based on information provided by mothers pertaining to family structure, parents' occupation, parents' education level, and parents' age at the birth of the child. According to teacher ratings, children who obtained a score of 1.5 standard deviations above the mean of their samegender classmates on the aggressiveness-disruptiveness and anxietywithdrawal scales or 1.5 standard deviations below the mean of their samegender classmates on the prosociality scale were classified as extremes on cach of these dimensions. These were termed the "extreme cases." Results showed that the number of extreme cases on the aggressivenessdisruptiveness scale in kindergarten was significantly related to FAI for both genders. Moreover, the number of extreme aggressive-disruptive cases who remained extreme after making the transition to grade one (i.e., stable cases) was also significantly related to FAl. For both genders, twice more stable aggressive-disruptive cases had a high FAI (i.e., top third) compared to transient extreme cases (i.e., extreme in kindergarten only). Discussion underlines the link between family adversity and incidence and persistence of behavioural problems during the early school years. It also stresses the need to consider family adversity to screen stable extreme aggressive-disruptive cases in kindergarten for early intervention purposes.
\end{abstract}

\title{
NOTES
}

1. En effet, dans l'étude de Vitaro, Desmarais-Gervais, Tremblay, et Gagnon (sous presse) réalisée auprès d'un vaste échantillon d'enfants québćcois, moins de un enfant sur quatre a fréquenté une garderie (ordinaire ou en milieu familial) plus de 10 heures par semaine en moyenne de lâge de 3 à 5 ans. 
2. Lorsque, par exemple, le père est absent de la famille, il arrive que l'emploi de celui-ci, son niveau d'éducation et son âge à la naissance de l'enfant de l'étude ne sont pas rapportés.

\section{RÉFÉRENCES}

Achenbach, T.M., \& Edelbrock, C.S. (1978). The classification of child psychopathology: A review and analysis of empirical efforts, Psychological Bulletin, 85, 1275-1301.

Achenbach, T.M., \& Edelbrock, C.S. (1981). Behavior problems and competencies reported by parents of normal and disturbed children aged four through sixteen. Monographs of the Society for Research in Child Development, 46(188).

Alexander, K.L., \& Entwistle, D.S. (1988). Achievement in the first two years of school: Patterns and process. Monographs of the Society for Research in Child Development, $53(218)$.

Behar, L.B., \& Stringfield, S. (1974). A behavior rating scale for the preschool child. Developmental Psychology, 10, 601-610.

Blishen, B.R., \& McRoberts, H.A. (1976). A revised socioeconomic index for occupations in Canada. Canadian Review of Sociology and Anthropology, 13, 71-79.

Bouchard, C. (1981). Perspectives écologiques de la relation parent(s)-enfant: Des compétences parentales aux compétences environnementales. Apprentissage ef Socialisation, 4, 4-23.

Boyle, M.H., \& Offord, D.R. (1988). Prevalence of childhood disorder, perceived need for help, family dysfunction and resource allocation for child welfare and children's mental health services in Ontario. Canadian Journal of Behavioural Sciences, 20, 374-388.

Campbell, S.B., \& Cluss, P. (1982). Peer relationships of young children with behavior problems. Dans R.H. Rubin \& H.S. Ross (Eds), Peer relationships and social skills in childhood (pp. 323-335). New York: Springer-Verlag.

Coleman, M.C., \& Gilliam, J.E. (1983). Disturbing behaviors in the classroom: A survey of teacher attitudes, Journal of Special Education, 17, 121-129.

Conners, C.K. (1969). A teacher rating scale for use in drug studies with children. American Journal of Psychiatry, 126, 884-888.

Dumas, J.E. (sous presse). Les désordres de la conduite chez l'enfant: Recensement et évaluation des travaux d'intervention en langue anglaise. Dans M. Provost \& R.E. Tremblay (Eds), Famille: Inadaptation et intervention. Nancy: Agence d'Arc.

Eron, L., \& Huesmann, L.R. (1983, juillet). The relation of prosocial behavior to the development of aggression and psychopathology, Communication au congres de l'International Society for the Study of Behavioral Development, Munich.

Fischer, M., Rolf, J.E., Hasazi, J.E., \& Cummings, L. (1984). Follow-up of a preschool epidemiological sample: Cross-age continuities and predictions of later adjustment with internalizing and externalizing dimensions of behavior. Child Development, 55, $137-150$.

Forehand, R., \& McMahon, R.G. (1981). Helping the noncompliant child. A clinician's guide to parent training. New York: Guilford Press.

Fowler, P.O., \& Park, R.M. (1979). Factor structure of the Preschool Behavior Questionnaire in a normal population. Psychological Reports, 45, 599-606.

Garbarino, J. (1982), Children and families in the social environment. New York: Adline.

Hoge, R.D., Meginbir, L., Khan, Y., \& Weatherall, D. (1985), Multitrait-multimethod analysis of the Preschool Behavior Questionnaire. Journal of Abnormal Child Psychology, 13, 119-127.

Kazdin, A.E. (1987). Treatment of antisocial behavior in children: Current status and future directions. Psychological Bulletin, 12, 187-203.

Kellam, S. G., Granch, J.D., Agrawal, K.C., \& Ensminger, M.E. (1975). Mental health and going to school. Chicago: University of Chicago Press. 
Kelso, J., \& Stewart, M.A. (1986). Factors which predict the persistence of aggressive con. duct disorder. Journal of Child Psychology and Psychiatry, 27, 77-86.

Kohn, M. (1977). The Kohn Social Competence Scale and Kohn Symptom Checklist for the preschool child: A follow-up report. Journal of Abnormal Child Psychology, 5, 249-263.

Laborit, H. (1979). L'Inhibition de l'action. Montréal: Presses de l'Université de Montréal.

Loeber, R. (1982). The stability of antisocial and delinquent child behavior: A review. Child Development, 53, 1431-1446.

Locber, R., Tremblay, R.E., Gagnon, C., \& Charlebois, P. (1989). Continuity and desistance in disruptive boys' early fighting at school. Development and Psychopathology, I, 39-50.

Markman, H.J., \& Jones-Leonard, D. (1985). Marital discord and children at risk: Implications for research and prevention. Dans W.H. Frankenburg, R.N. Emde, \& J.W. Sullivan (Eds), Early identification of children at risk (pp. 59-77). New York: Plenum.

Masters, R.D. (1979). Beyond reductionism: Five basic concepts in human ethology. Dans M. Von Cranach (Ed), Human ethology-Claims and limits of a new discipline (pp. 265-284). Cambridge: Cambridge University Press.

McDermott, P.Q., \& Watkins, M.W. (1981). Dimensions of maladaptive behavior among kindergarten level children. Behavioral Disorders, 7, 11-17.

Meijer, C.J.W., \& Foster, S.F. (1989). The effect of teacher self-efficacy on referral chance. Journal of Special Education, 22, 378-385.

Milich, R., \& Landau, S. (1984). A comparison of the social status and social behavior of aggressive and aggressive-withdrawn boys. Journal of Abnormal Child Psychology, 12, 277-288,

Milich, R., Loney, J., \& Landau, S. (1982). Independent dimensions of hyperactivity and aggression: A validation with playroom observation data. Journal of Abnormal Psychology, 91, 183-198.

Ministère de l'Education du Québec. (1983). Principes de l'education préscolaîre. Québec: Auteur.

O'Donnell, J., Leicht, D.J., Phillips, F.L., Marnett, J.P., \& Horn, W.F. (1988). Stability of children's behavior problems: A 3-1/2-year longitudinal study. Journal of Applied Developmental Psychology, 9, 233-241.

Olweus, D. (1984). Development of stable aggressive reaction patterns in males. Dans R. Blanchard \& C. Blanchard (Eds), Advances in aggression research, vol. I (pp. 103-137). New York: Academic Press.

Parker, J.G., \& Asher, S.R. (1987). Peer relations and later personal adjustment: Are low accepted children at risk? Psychological Bulletin, 102, 357-389.

Patterson, G.R. (1982). Coercive family process. Eugene: Castalia.

Patterson, G.R., Cobb, J.A., \& Ray, R.S. (1983). A social engineering technlogy for retraining the families of aggressive boys. Dans H.E. Adams \& J.P. Unikel (Eds), Issues and trends in behavior therapy (pp. 139-210). Springfield, IL: Charles C. Thomas.

Patterson, G.R., Reid, J.B., Jones, R.R., \& Conger, R.W. (1975). A social learning approach to family intervention, vol. 1. Eugene: Castalia.

Quay, H.C. (1979). Classification. Dans H.C. Quay \& J.S. Werry (Eds), Psychopathological disorders of childhood (pp. 1-42). New York: John Wiley \& Sons,

Quinton, D., \& Rutter, M. (1976). Early hospital and later disturbances of behavior: An attempted replication of Douglas' findings. Developmental Medicine and Child Neurology, 18, 447-459.

Robins, L.N. (1966). Deviant children grown up. Baltimore: Williams \& Wilkins.

Robins, L.N. (1986). The consequences of conduct disorder in girls. Dans D. Olweus, J. Block, \& M. Radke-Yarrow (Eds), Development of antisocial and prosocial behavior (pp. 385-414). New York: Academic Press. 
Ross, A.O., Lacey, H.M., \& Parton, D.A. (1965). The development of a behavior checklist for boys. Child Development, 36, 1013-1027.

Rubin, K.H., \& Clark, M.L. (1983). Preschool teachers' ratings of behavioral problems: Observational, sociometric, and social-cognitive correlates. Joturnal of Abnormal Child Psychology, 11, 273-286.

Rubin, K.H., Moller, L., \& Emptage, A. (1986). The Preschool Behaviour Questionnaire; A useful index of behaviour problems in elementary school-age children? Canadian Journal of Behavioural Sciences, 19, 86-100.

Rutter. M. (1967). A children's behavior questionnaire for completion by teachers: Preliminary findings. Journal of Child Psychology and Psychiatry, 8, 1-11.

Rutter, M. (1985). Resilience in the face of adversity: Protective factors and resistance to psychiatric disorders. British Journal of Psychiatry, 147, 598-611.

Rutter, M., Cox, A., Tupling, C., Berger, M., \& Yule, W. (1975). Attainment and adjustment in two geographical areas. 1. The prevalence of psychiatric disorder. British Journal of Psychiatry, 126, 493-509.

Rutter, M., Tizard, J., \& Withmore, K. (1970). Education, health and behaviour. New York: Robert E. Krieber.

Schachar, R., Rutter, M., \& Smith, A. (1981). The characteristics of situationally and pervasively hyperactive children: Implications for syndrome definition. Journal of Child Psychology and Psychiatry, 22, 375-392.

Schachar, R., \& Wachsmuth, R. (1991). Family dysfunction and psychosocial adversity: Comparison of attention deficit disorder, conduct disorder, normal and clinical controls, Canadian Journal of Behavioural Sciences, 23, 332-348.

Schneider, B., Boucher, C., Rivest, C., \& Taillefer, S. (1984). La présence du stress chez l'enfant en rapport avec son niveau ultérieur d'adaptation: Quels sont les enfants les moins vulnérables? Apprentissage et Socialisation, 7, 208-218.

Tremblay, R.E. (1991). Reproduction sociale de l'inadaptation: Le cas des comportements agressifs et antisociaux. Dans M. Provost \& R.E. Tremblay (Eds), Famille: Inadaptation et intervention (pp. 107-140). Nancy: Agence d'Arc.

Tremblay, R.E., \& Baillargeon, L. (1984). Les difficultés de comportements d'enfants immigrants dans les classes d'accueil au préscolaire. Revue Canadienne de l'Education. 9, 154170 .

Tremblay, R.E., Desmarais-Gervais, L., Gagnon, L., \& Charlebois, P. (1987). The Preschool Behaviour Questionnaire: Stability of its factor structure between cultures, sexes, ages and socioeconomic classes. International Journal of Behavioural Development, 10, 467-484.

Tremblay, R.E., Loeber, R., Gagnon, C., Charlebois, P., Larivée, S., \& Le Blanc, M. (1991). Disruptive boys with stable and unstable high fighting behavior patterns during junior elementary school. Journal of Abnormal Child Psychology, 19, 285-300.

Tremblay, R.E., Masse, B., Perron, D., Le Blanc, M., Schwartzman, A.E., \& Ledingham, J.E. (1992). Early disruptive behavior, poor school achievement, delinquent behavior, and delinquent personality: Longitudinal analyses. Journal of Consulting and Clinical Psychology, 60, 64-72.

Tremblay, R.E., Vitaro, F., Bertrand, L., Le Blane, M., Beauchesne, H., Boileau, H., \& David, L. (sous presse). Parent and child training to prevent early onset of delinquency: The Montréal-longitudinal-experimental study. Dans J. McCord \& R.E. Tremblay (Eds), Preventing deviant behavior from birth to adolescence: Experimental approaches. New York: Guilford Press.

Tremblay, R.E., Vitaro, F., Gagnon, C., Piché, C., \& Royer, N. (sous presse). A prosocial scale for the Preschool Behavior Questionnaire. International Journal of Behavioural Development.

Tremblay, R.E., Vitaro, F., Gagnon, C., \& Perron, D. (1989, juin), La stabilité temporelle 
et situationnelle des comportements turbulents chez les enfants: Contribution des évaluations par les mères ef les enseignants. Communication au congrès annuel de la Société canadienne de psychologie, Halifax.

Tremblay, R.E., \& Zhou, R.-M. (1991). Le dépistage des difficultés d'adaptation sociale chez les garçons de milieux socioéconomiques faibles: De la maternelle a la fin de l'école primaire. Rapport de recherche au CQRS et au FCAR, Groupe de recherche sur l'adaptation psychosociale chez l'enfant, Université de Montréal.

Trites, R.L., \& Laprade, K. (1983). Evidence for an independent syndrome of hyperactivity. Child Psychology and Psychiatry, 24, 573-586.

Vitaro, F., Desmarais-Gervais, L., Tremblay, R.E., \& Gagnon, C. (sous presse). Difficultés d'adaptation sociale en début de scolarisation et milieu de garde à la période préscolaire. Revue des Sciences de l'Education.

Watt, N.F., Anthony, J., Wynne, L.C., \& Rolf, J.E. (1984). Children at risk for schizophrenia. A longitudinal perspective. Cambridge: Cambridge University Press.

Weir, K., \& Duveen, G. (1981). Further development and validation of the Prosocial Behaviour Questionnaire for use by teachers, Journal of Child Psychology and Psychiatry, 22, 357-374.

Werner, E.E., \& Smith, R.S. (1982). Vulnerable but invincible: A longitudinal study of resilient children and youth. Montréal: McGraw-Hill. 